\title{
Factores asociados a la nefropatía inducida por contraste en pacientes hospitalizados a quienes se les realizó tomografía axial computarizada
}

\section{Factors associated with contrast-induced nephropathy in hospitalized patients who underwent computed tomography}

- Maickol Jhoel Tirado Castro ${ }^{1,2^{*}}, \odot$ Liliana Rocío Cataño Vargas ${ }^{1,2,3}, \odot$ Franklyn Edwin Prieto Alvarado ${ }^{4}$

\author{
${ }^{1}$ Grupo Epidemioloía y Salud Pública de la región Surcolombiana, Maestría en epidemiología, Ciencias de la saluNeivad, Universidad Surcolombiana, Neiva, Colombia \\ ${ }^{2}$ Grupo de investigación y Epidemiología y Salud Pública de la región Surcolombiana, Grupo Epidemiología y Salud Pública de la región Surcolombiana, Maestría en \\ epidemiología, Ciencias de la saluNeivad, Universidad Surcolombiana, Neiva, Colombia. \\ ${ }^{3}$ Servicio de Nefrología, Clínica Medilaser S. A. Neiva, Colombia.
}

${ }^{4}$ Salud pública, departamento Maestría en epidemiología, Facultad de Ciencias de la salud, Universidad Surcolombiana, Neiva, Colombia.

\begin{abstract}
Resumen
Introducción: la nefropatía inducida por medio de contraste es la tercera causa de insuficiencia renal aguda en pacientes hospitalizados. En múltiples estudios se ha observado que un factor importante para su desarrollo es el uso de medio de contraste vía intra-arterial y en las angiografías en general. Nuestro objetivo es identificar los factores asociados a nefropatía inducida por contraste en pacientes hospitalizados, a quienes se les realizó tomografía axial computarizada con medio de contraste yodado en una institución de salud de alta complejidad de Neiva, Colombia, durante el 2016.

Materiales y métodos: se desarrolló un estudio de casos y controles no pareado con 108 pacientes, 36 casos y 72 controles hospitalizados llevados a tomografía axial computarizada diagnóstica o terapéutica con medio de contraste radiológico yodado.

Resultados: los factores asociados con nefropatía inducida por medio de contraste encontrados en los pacientes hospitalizados llevados a TAC contrastada fueron, el sexo (hombre) $(\mathrm{OR}=3,22 ; \mathrm{IC}=95 \% 1,33-7,76 ; \mathrm{p}=0,009)$, y el servicio de procedencia (hospitalización en sala general) $(\mathrm{OR}=0,26 ; \mathrm{IC}=95 \% 0,07-1,00 ; \mathrm{p}=0,051)$.

Palabras clave: Insuficiencia renal aguda, tomografía computarizada, medio de contraste, factores de riesgo.
\end{abstract}

doi: http://dx.doi.org/10.22265/acnef.0.0.303

\begin{abstract}
Introduction: Contrast-induced Nephropathy is the third most common cause of acute renal failure in patients. It has been observed in several studies that an important factor is the usage of a contrast given via IV and the angiograms. Our goal is to identify the factors associated to a Contrast-induced Nephropathy in patients who had a computarized axial tomography through an iodine contrast in a health institution of high complexity in Neiva, Colombia during 2016.

Material and methods: A cased study and no-matched tests were applied in 108 patients (36 cases and 76 tests), who had a computarized axial dianostic or therapeutic tomography through an iodine radiological contrast.

Results: The factors associated to contrast-induced Nephropathy, through a contrast dye, found in patients who had a CAT were the gender (male) $(\mathrm{OR}=3,22 ; \mathrm{CI}=95 \% 1,33-7,76 ; \mathrm{p}=0,009)$ and the place where the procedure was made (hospitalization in general wards $)(\mathrm{OR}=0,26$; $\mathrm{CI}=95 \% 0,07-1,00 ; \mathrm{p}=0,051)$.
\end{abstract}

Key words: Acute kidney injury, computed tomograpy, contrast media, risk factors.

doi: http://dx.doi.org/10.22265/acnef.0.0.303

Citación: Tirado-Castro MJ, Cataño-Vargas LR, Prieto-Alvarado FE. Factores asociados a la nefropatía inducida por contraste en pacientes hospitalizados a quienes se les realizó tomografía axial computarizada. Rev. Colomb. Nefrol. 2018;5(2):118-126. doi: http://dx.doi.org/10.22265/acnef.0.0.303

*Correspondencia: Maickol Jhoel Tirado Castro, tiradotrabalho@gmail.com

Recibido: $10.3 .18 \cdot$ Aceptado: 8.6.18 Publicado en línea: 9.8 .18 


\section{Introducción}

$\mathrm{S}$ e estima que cada año en el mundo, cerca de dos millones de personas mueren a causa de la insuficiencia renal aguda y aquellos que sobreviven tienen un mayor riesgo de desarrollar enfermedad renal crónica. Estudios de insuficiencia renal aguda hospitalaria en países desarrollados muestran que esta complicación genera entre 3,2 y 9,6 \% de las hospitalizaciones, y tiene una mortalidad del $20 \%$, y hasta $50 \%$ en los pacientes de Unidad de Cuidado Intensivo ${ }^{1,2,3}$.

La nefropatía inducida por medio de contraste1 ha sido reportada como la tercera causa de insuficiencia renal aguda intrahospitalaria ${ }^{3,4,5,6,7}$. En algunos países como Estados Unidos, los medios de contraste radiológico yodados se utilizan anualmente en más de diez millones de procedimientos, y se estima que la probabilidad de desarrollar nefropatía inducida por medio de contraste (NIC) en una persona que tenga uno o más factores de riesgo es de 20,7 $\%-23,3 \%{ }^{8}$. Este riesgo es más de diez veces mayor que el de una persona sin enfermedad de base $(1,5$ $\%-2 \%)^{3}$.

En Latinoamérica, Da Silva reportó que la incidencia de NIC después de una tomografía computarizada es de 3,75\%-15,75\%?․ Los estudios han demostrado asociación entre NIC y resultados clínicos adversos, tales como las complicaciones cardiovasculares, el requerimiento de diálisis y la muerte $\frac{4,6,8}{}$.

Las guías de prevención de NIC han sido elaboradas con pacientes de cardiología intervencionista, población que difiere de los pacientes llevados a tomografía. Por esta razón, los factores de riesgo deben ser identificados y valorados en el grupo de pacientes a quienes se les va a realizar un procedimiento diagnóstico o terapéutico con medios de contraste radiológicos yodados $s^{14}$. Identificar pacientes en riesgo de NIC será útil en el momento de la toma de decisiones clínicas para disminuir la incidencia de la enfermedad, en la optimización de recursos y en la focalización de acciones preventivas.

\section{Materiales y métodos}

Se llevó a cabo un estudio de casos y controles no pareado en pacientes hospitalizados en los servicios de observación, urgencias y hospitalización (general y UCI), a quienes se les realizó tomografía axial computarizada diagnóstica o terapéutica con medio de contraste radiológico yodado. El estudio fue desarrollado en una institución de salud de III/IV nivel de complejidad del sector privado, referente en la región surcolombiana, que atiende a usuarios del sistema de salud en Neiva, Colombia, durante el 2016.

Se trabajó con el censo de la población (figura 1), por agotamiento muestral hasta encontrar casos (muestreo por incidencia acumulada), y se buscó un escenario de controles que fuera plausible 1:2. De acuerdo con lo anterior, con un nivel de confianza del $90 \%$, el poder calculado basado en el método de aproximación normal fue del $80 \%$.

Tipo de contraste: según el protocolo institucional, el medio de contraste fue administrado por vía intravenosa y la dosis no fue variable.

Definición de caso: paciente hospitalizado a quien se le realizó TAC contrastada y que $48,72 \mathrm{o}$ 120 horas después reportó un incremento relativo de la creatinina sérica mayor al $25 \%$ o un aumento absoluto mayor o igual a $0,5 \mathrm{mg} / \mathrm{dL}$ con respecto a su valor previo a la tomografía.

Definición de control: paciente hospitalizado llevado a TAC contrastada que 48, 72 o 120 horas después no desarrolló el evento de estudio.

Criterios de inclusión: pacientes llevados a una TAC con medio de contraste radiológico yodado, mayores de 18 años, hospitalizados en sala general, UCI y en sala de observación de urgencias.

Criterios de exclusión: pacientes a quienes se les realizó un procedimiento de cardiología intervencionista $y / 0$ que recibieron otra dosis de medio de contraste en menos de 72 horas.

Para el análisis de los datos se utilizó medidas de tendencia central, dispersión, proporciones y 
razones, según el tipo de variable. La medida de asociación epidemiológica utilizada fue el $\mathrm{Odds}$ Ratio y se empleó el modelo de regresión logística binario multivariado utilizando el método de introducir variables (Enter) a partir de criterios de plausibilidad y evidencia previa. Para llevar a cabo los análisis, se utilizó el paquete estadístico STATA 14.0.
Esta investigación fue clasificada sin riesgo según la resolución 8430 de 1993, se tuvo en cuenta los principios éticos establecidos en el Reporte Belmont y se obtuvo el consentimiento informado del custodio de la historia clínica.

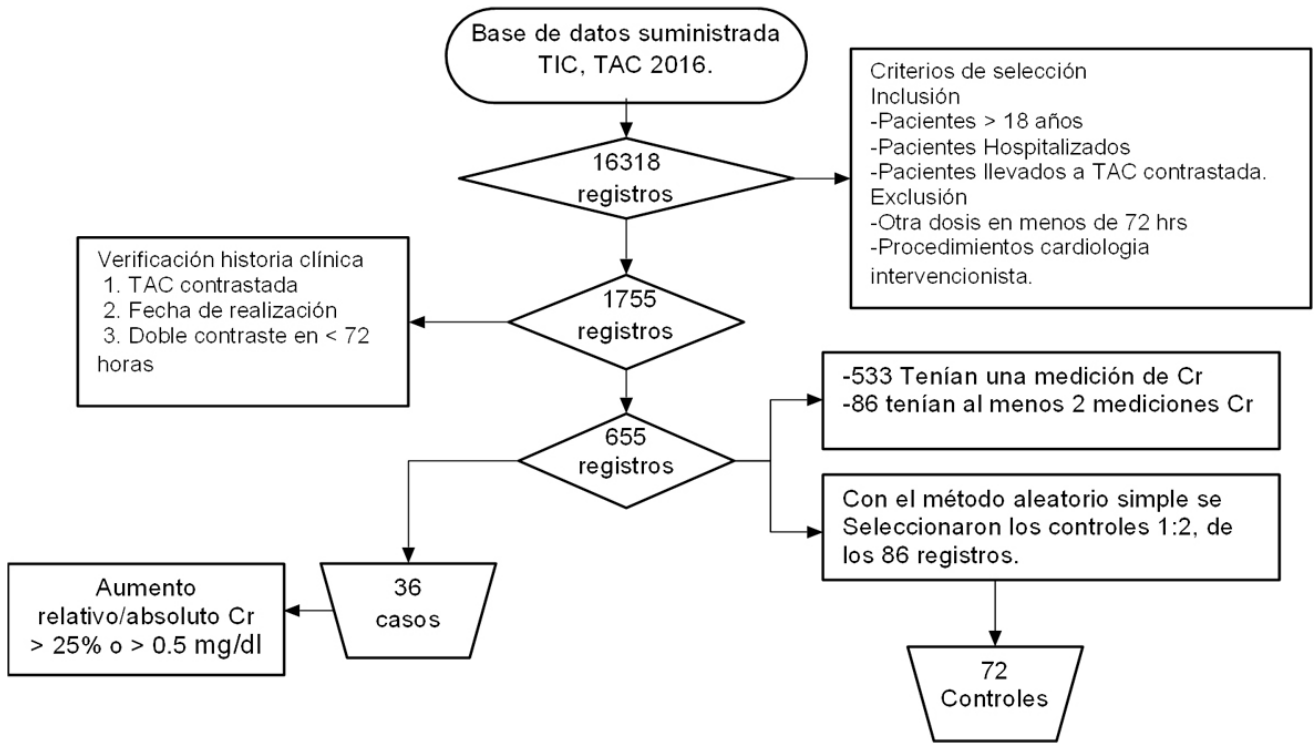

Figura 1. Selección de pacientes hospitalizados llevados a TAC contrastada, Neiva, Colombia, 2016.

\section{Resultados}

Durante el 2016, fueron llevados a tomografía contrastada 655 pacientes hospitalizados en una institución de alta complejidad de Neiva, Colombia. Entre ellos, 122 (18,6 \%) tenían, al menos, dos mediciones de creatinina, una anterior al procedimiento y otra a las 48-72 horas, o hasta el quinto día. Esto permitió la configuración de los casos (36) y la selección de los controles (72). El tipo de TAC más frecuente fue el de abdomen $(72,2$ $\%)$.

Las características demográficas y clínicas de los 108 pacientes son presentadas en la tabla 1. La incidencia de NIC1 fue de 5,5\%. No se encontraron diferencias entre casos y controles, excepto en la variable sexo $(p=0,0140)$.

Al explorar la relación de NIC con las características demográficas y clínicas de interés reportadas por la literatura (tabla 2), se mantuvo la asociación con la variable sexo $(\mathrm{OR}=2,8 ; \mathrm{IC}=95$ $\%$ 1,12 - 7,11, p=0,0143). Sin embargo, se encontró también asociación no significativa entre NIC y edad, hematocrito, PAM, TFGe, diabetes, ICC y nefroprotección.

Al ajustar los posibles factores de confusión (tabla 3), la NIC se encontró asociada con el sexo y el servicio de procedencia (hospitalización en sala general). 
Rev. Colomb. Nefrol. 2018;5(2): 118 - 126 http://www.revistanefrologia.org http://dx.doi.org/10.22265/acnef.0.0.303

Tabla 1.

Características demográficas y clínicas de los pacientes hospitalizados llevados a TAC contrastada, Neiva, Colombia, 2016.

\begin{tabular}{|c|c|c|c|c|c|}
\hline \multicolumn{6}{|c|}{ Características demográficas } \\
\hline & $\begin{array}{c}\text { NIC positivo } \\
\mathrm{n}=36\end{array}$ & $\%$ & $\begin{array}{c}\text { NIC negativo } \\
\mathrm{n}=72\end{array}$ & $\%$ & valor de $\mathrm{p} \alpha$ \\
\hline Hombre & 24 & 66,7 & 30 & 41,7 & 0,0140 \\
\hline Edad $\geq 65$ años & 15 & 41,7 & 26 & 36,1 & 0,5750 \\
\hline $18-45$ & 6 & 16,7 & 22 & 30,6 & 0,3600 \\
\hline $46-59$ & 7 & 19,4 & 16 & 22,2 & \\
\hline $60-74$ & 13 & 36,1 & 18 & 25,0 & \\
\hline$>74$ & 10 & 27,8 & 16 & 22,2 & \\
\hline \multicolumn{6}{|c|}{ Características clínicas } \\
\hline & $\begin{array}{c}\text { NIC positivo } \\
\mathrm{n}=36\end{array}$ & $\%$ & $\begin{array}{c}\text { NIC negativo } \\
n=72\end{array}$ & $\%$ & valor de $\mathrm{p} \alpha$ \\
\hline Urgencias & 21 & 58,3 & 31 & 43,1 & 0,2360 \\
\hline Hospitalización & 4 & 11,1 & 16 & 22,2 & \\
\hline UCI* & 25 & 34,7 & 11 & 30,6 & \\
\hline $\mathrm{Hb}$ anormal** & 28 & 77,8 & 55 & 76,4 & 0,8720 \\
\hline Hematocrito $<34$ & 18 & 50,0 & 28 & 38,9 & 0,2710 \\
\hline $\mathrm{PAM}^{* * *}<70 \mathrm{mmhg}$ & 3 & 8,3 & 2 & 2,8 & 0,1950 \\
\hline $\mathrm{TFGe}+<60 \mathrm{ml} / \mathrm{min} / 1,73 \mathrm{~m} 2$ & 11 & 34,4 & 21 & 31,8 & 0,8000 \\
\hline Cr_prec $++\geq 1,5$ & 4 & 11,1 & 9 & 12,5 & 0,8340 \\
\hline $\mathrm{Hb}$ & 11,2 & $\pm 1,71$ & 11,56 & $\pm 2,38$ & 0,4112 \\
\hline Hematocrito & 34,54 & $\pm 5,40$ & 35,37 & $\pm 7,33$ & 0,5492 \\
\hline PAM & 84,42 & $\pm 9,84$ & 87,93 & $\pm 10,99$ & 0,1082 \\
\hline TFGe & 88,57 & $\pm 82,32$ & 72,39 & $\pm 42,55$ & 0,1801 \\
\hline Cr_pre & 0,97 & $\pm 0,55$ & 1,22 & $\pm 1,081$ & 0,1798 \\
\hline \multicolumn{6}{|c|}{ Comorbilidades } \\
\hline & $\begin{array}{c}\text { NIC positivo } \\
\mathrm{n}=36\end{array}$ & $\%$ & $\begin{array}{c}\text { NIC negativo } \\
n=72\end{array}$ & $\%$ & valor de $\mathrm{p} \alpha$ \\
\hline Diabetes & 7 & 19,4 & 7 & 9,7 & 0,156 \\
\hline Hipertensión & 9 & 25,0 & 17 & 23,6 & 0,874 \\
\hline $\mathrm{ICC}+++$ & 3 & 8,3 & 3 & 4,2 & 0,373 \\
\hline Cáncer & 7 & 19,4 & 13 & 18,1 & 0,861 \\
\hline \multicolumn{6}{|c|}{ Antecedentes farmacológicos } \\
\hline & $\begin{array}{c}\text { NIC positivo } \\
\mathrm{n}=36\end{array}$ & $\%$ & $\begin{array}{c}\text { NIC negativo } \\
\mathrm{n}=72\end{array}$ & $\%$ & valor de $p \alpha$ \\
\hline Nefrotóxicos & 6 & 16,7 & 18 & 25,0 & 0,326 \\
\hline Nefroprotección & 23 & 63,9 & 50 & 69,4 & 0,561 \\
\hline
\end{tabular}

$\alpha$ Pruebas de chi cuadrado, de Pearson o t-student para diferencia de medias.

*UCI: unidad de cuidado intensivo

**Hb: hemoglobina $\mathrm{H}$ : hombre $<13,5$; M: mujer $<12,6$

***PAM: presión arterial media

+TFGe: tasa de filtración glomerular estimada (32 casos/66 controles)

++ Cre prec : creatinina previa

+++ ICC: insuficiencia cardiaca congestiva 
Rev. Colomb. Nefrol. 2018;5(2): 118 - 126 http://www.revistanefrologia.org http://dx.doi.org/10.22265/acnef.0.0.303

Tabla 2.

Factores relacionados con nefropatía inducida por contraste en pacientes hospitalizados llevados a TAC contrastada, Neiva, Colombia, 2016.

\begin{tabular}{ccc} 
Variables & $\begin{array}{c}\text { OR crudo }(\mathrm{IC}=95 \%) \\
\mathrm{n}=108\end{array}$ & Valor de $\mathrm{p} \alpha$ \\
Características demográficas & & \\
\hline Sexo/Hombre & $2,8(1,12-7,11)$ & 0,0143 \\
Edad $\geq 65$ años & $1,2(0,51-3,08)$ & 0,5749 \\
\hline Características Clínicas & & \\
\hline Hb anormal* & $1,0(0,38-3,26)$ & 0,8718 \\
Hematocrito $<34$ & $1,5(0,64-3,80)$ & 0,2710 \\
PAM**<70 mmhg & $3,1(0,34-39,32)$ & 0,1952 \\
Cr+ $\geq 1,5 \mathrm{mg} / \mathrm{dl}$ & $0,8(0,18-3,44)$ & 0,8344 \\
TFGe++<60 ml/min/1,73m2 & $1,1(0,40-2,97)$ & 0,8002 \\
\hline Antecedentes - Comorbilidades & & 0,1562 \\
Diabetes & $2,2(0,60-8,19)$ & 0,8736 \\
Hipertensión & $1,0(0,37-2,96)$ & 0,3729 \\
ICC+++ & $2,0(0,26-16,34)$ & 0,8610 \\
Cáncer & $1,0(0,33-3,34)$ & 0,3261 \\
Nefrotóxicos & $0,6(0,17-1,80)$ & 0,5609 \\
\hline Nefroprotección & $1,2(0,50-3,22)$ & \\
\hline
\end{tabular}

$\alpha$ Prueba chi cuadrado, de Pearson

*Hb: Hemoglobina H: Hombre <13,5; M: Mujer $<12,6$

**PAM: presión arterial media

$+\mathrm{Cr} \geq 1,5$ : Creatinina previa $\geq 1,5 \mathrm{mg} / \mathrm{dl}$

++TFGe: Tasa de Filtración Glomerular estimada por Cockroft y Gault (n=98)

+++ ICC: Insuficiencia Cardiaca Congestiva

Tabla 3.

Factores asociados con nefropatía inducida por contraste en pacientes hospitalizados llevados a TAC contrasta. Neiva-Colombia 2016.

\begin{tabular}{cccc} 
Covariable candidata & OR Crudo $(\mathrm{IC}=95 \%)$ & OR ajustado $(\mathrm{IC}=95 \%)$ & Valor $\mathrm{p}$ \\
Hombre & $2,8(1,12-7,11)$ & $3,22(1,33-7,76)$ & 0,009 \\
DM & $2,2(0,60-8,19)$ & $3,48(0,96-12,57)$ & 0,057 \\
Hospitalización++ & $0,36(0,079-1,38)$ & $0,26(0,07-1,00)$ & 0,051 \\
UCI++ & $0,65(0,24-1,74)$ & $0,58(0,22-1,49)$ & 0,258 \\
\hline
\end{tabular}

Probabilidad: chi2=0,0104; Log likelihood=-62,155407; Pseudo R2=0,0958

++ Categoría de referencia: urgencias

122 Factores asociados a nefropatía inducida por contraste. 


\section{Discusión}

Los medios de contraste yodados son comúnmente utilizados en estudios diagnósticos o terapéuticos, con el propósito de visualizar vasos, tejidos y órganos. Usualmente su uso es seguro, pero la $\mathrm{NIC}^{1}$ ha sido descrita como un evento adverso que ocasiona aumento de morbimortalidad en quienes lo desarrollan ${ }^{2}$.

La literatura reporta un gran número de estudios $\frac{1,3.4}{3}$ sobre el desarrollo de esta complicación, sus factores de riesgo y sus desenlaces adversos luego de la administración intraarterial del medio de contraste. Sin embargo, pocos estudios exploran estas relaciones en pacientes hospitalizados que reciben medio de contraste intravenoso.

El procedimiento de recolección de datos para el reclutamiento de los casos se realizó, en verificación simultánea, a partir de la revisión de los registros de laboratorios (creatininas pre y post, según criterios), de los registros de lecturas de tomografías contrastadas, de la base de datos con los códigos de las TAC realizadas durante 2016, de la historia clínica sistematizada y de los registros de enfermería. Esto permitió garantizar la calidad de los datos y controlar los posibles sesgos de información.

La incidencia de NIC posterior a la realización de TAC con medio de contraste yodado fue de $5,5 \%$, resultado consistente con estudios de NIC reportados por otros autores donde la incidencia varía entre 3,4 y 13,9\%, según la definición de caso utilizada en el presente estudio ${ }^{5,6,7,8}$.

La diabetes ha sido descrita como un factor independiente para el desarrollo de esta complicación $\frac{13}{3}$. Los cambios crónicos túbulointersticiales hacen particularmente susceptible al riñón en la intensificación de la hipoxia y al estrés oxidativo posterior a la administración del medio de contraste. Aunque la asociación entre NIC y diabetes no fue estadísticamente significativa, este antecedente ha sido reportado como uno de los principales factores de riesgo para el desarrollo de NIC, así como la edad avanzada ( $>65$ años) y la falla renal previa $7,9 \cdot 10,11$.
Llama la atención la asociación del sexo (masculino) como predictor del desarrollo de NIC $(\mathrm{OR}=3,22 ; \mathrm{IC}=95 \%$ 1,33 - 7,76; $\mathrm{p}=0,009)$, hallazgo concordante con el estudio de Inga Skarupskiene et al. $\stackrel{14}{ }$. En estudios realizados en Centroamérica, se ha reportado mayor prevalencia de enfermedad renal túbulointersticial en el sexo masculino $(78,3 \%)$ relacionada con factores medioambientales como el trabajo agrícola, la exposición a agroquímicos y la deshidratación (sudoración excesiva) $)^{15,17}$. En Colombia, según el informe de la cuenta de alto costo $2015 \frac{16}{6}$, la prevalencia de la terapia de reemplazo renal en hombres es 89,2 por cada 100.000 habitantes (en las mujeres es de 56,6 por 100.000 habitantes). Estos datos concuerdan con la experiencia institucional, según el departamento de estadística, donde la mayoría de los pacientes con insuficiencia renal crónica atendidos son hombres (65 \%) procedentes del sur del departamento de Huila, donde la base de la economía son las actividades agrícolas.

En relación con el servicio de procedencia, la estancia hospitalaria en sala general se comportó como un factor protector $(\mathrm{OR}=0,26 ; \mathrm{IC}=95 \% 0,07$ - 1,$00 ; p=0,05)$ cuando se comparó con pacientes procedentes del servicio de urgencias o la unidad de cuidados intensivos $\frac{11}{1}$. Este resultado puede estar relacionado con la evaluación del riesgo previo y posterior a la exposición a la tomografía axial computarizada contrastada, lo que permitiría tomar decisiones clínicas para prevenir la NIC en este grupo de pacientes, pues los pacientes en sala general difieren de los pacientes en UCI y urgencias en sus características clínicas y estado patológico.

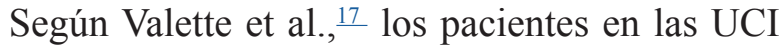
reportan incremento del riesgo de $\mathrm{NIC}^{18}$. Sin embargo, estos resultados deben ser interpretados con cuidado, debido a que es probable que los cambios en la creatinina puedan ser atribuibles a factores distintos de la exposición al contraste $\frac{10,19}{}$, tales como el estado metabólico, sepsis o exposición a nefrotóxicos. Por lo tanto, la incidencia de NIC puede ser sobreestimada. 
De acuerdo con los hallazgos presentados, se debe insistir en la valoración del riesgo y seguimiento de los pacientes con antecedentes de diabetes mellitus tipo 2, pacientes hospitalizados en sala de observación urgencias y en unidades de cuidados intensivos, pues la exposición al medio de contraste es cada vez más frecuente en la práctica clínica: es utilizado como ayuda diagnóstica para imagenología y procedimientos endovasculares o de cardiología intervencionista. Por lo anterior, identificar pacientes en riesgo de desarrollar NIC será útil en el momento de la toma de decisiones clínicas para disminuir la incidencia de esta enfermedad, enfocar los esfuerzos en la realización de acciones preventivas y optimizar recursos.

Este estudio presenta algunas limitaciones. Fue desarrollado en un único centro hospitalario, pero la variabilidad en la práctica clínica entre los profesionales al interior de la institución eventualmente podría equipararse a la variabilidad en la práctica clínica entre las instituciones. Además, el seguimiento de la función renal a través de pruebas de control postexposición al medio de contraste no se realizaba rutinariamente a los pacientes en la institución, por lo que no se encontraron mediciones de creatinina en todos los pacientes llevados a TAC contrastada, lo cual pudo subestimar la incidencia de la nefropatía asociada a medio de contraste. Por último, el uso de nefroprotección no se encontraba estandarizado, razón por la cual no fue posible medir el impacto de esta variable en el desarrollo de NIC.

\section{Conclusiones}

Durante el 2016, fueron llevados a TAC contrastada 655 pacientes, de los cuales a $18,6 \%$ se les realizó seguimiento de creatinina postexposición a las 48, 72 o 120 horas.

A pesar de que el seguimiento de la función renal a través de pruebas de control postexposición al medio de contraste no se realizaba rutinariamente en la institución, la incidencia de nefropatía inducida por medio de contraste en los pacientes hospitalizados llevados a TAC contrastada durante el 2016 fue de $5,5 \%$.
Los factores asociados con nefropatía inducida por medio de contraste encontrados en los pacientes hospitalizados llevados a TAC contrastada durante el 2016, fueron el sexo (hombre) $(\mathrm{OR}=3,22 ; \mathrm{IC}=95$ $\%$ 1,33 - 7,76; $p=0,009)$, y el servicio de procedencia (hospitalización en sala general) $(\mathrm{OR}=0,26 ; \mathrm{IC}=95$ $\% 0,07-1,00 ; \mathrm{p}=0,051)$.

\section{Conflicto de interés}

Los autores declaran que no existe ningún conflicto de interés.

\section{Financiación}

Por medio de este escrito, manifestamos no haber recibido financiación de ningún tipo durante el desarrollo y finalización del artículo.

\section{Responsabilidades éticas}

\section{Protección de personas y animales}

Los autores declaran que para esta investigación no se han realizado experimentos en seres humanos ni en animales.

\section{Confidencialidad de los datos}

Los autores declaran que han seguido los protocolos de su centro de trabajo sobre la publicación de datos de pacientes.

\section{Derecho a la privacidad y consentimiento informado}

Los autores declaran que en este artículo no aparecen datos de pacientes.

\section{Aporte de los autores}

Anteproyecto, proyecto, recolección y análisis de datos:

Maickol Jhoel Tirado Castro, estudiante maestría en epidemiología

Liliana Rocío Cataño Vargas, estudiante maestría en epidemiología

Tutor metodológico y estadístico:

Franklyn Edwin Prieto Alvarado, médico especialista en epidemiología, magister en salud pública y bioética, $\mathrm{PhD}$ en salud pública. 


\section{Rev. Colomb. Nefrol. 2018;5(2): 118 - 126 http://www.revistanefrologia.org}

http://dx.doi.org/10.22265/acnef.0.0.303

\section{Referencias}

1. Dangas G, Iakovou I, Nikolsky E, Aymong ED, Mintz GS, Kipshidze NN, et al. Contrast-induced nephropathy after percutaneous coronary interventions in relation to chronic kidney disease and hemodynamic variables. Am J Cardiol. 2005;95(1):13-19. https:// doi.org/10.1016/j.amjcard.2004.08.056

2. Meinel FG, De Cecco CN, Schoepf UJ, Katzberg R. Contrast-induced acute kidney injury: definition, epidemiology, and outcome. BioMed Research International. 2014;2014. https://doi.org/10.1155/2014/859328

3. Andreucci M, Solomon R, Tasanarong A. Side effects of radiographic contrast media: pathogenesis, risk factors, and prevention. BioMed Research International. 2014;2014. https://doi.org/10.1155/2014/741018

4. Bartholomew BA, Harjai KJ, Dukkipati S, Boura JA, Yerkey MW, Glazier S, et al. Impact of nephropathy after percutaneous coronary intervention and a method for risk stratification. American J Cardiol. 2004;93(12):1515-1519. https://doi.org/10.1016/j. amjcard.2004.03.008

5. Mehran R, Nikolsky E. Contrast-induced nephropathy: definition, epidemiology, and patients at risk. Kid Int Suppl. 2006;69:S11-S15. https://doi.org/10.1038/sj.ki.5000368

6. Andreucci M, Solomon R, Tasanarong A. Side Effects of Radiographic Contrast Media: Pathogenesis, Risk Factors, and Prevention. BioMed Res Int. 2014;2014. https://doi.org/10.1155/2014/741018

7. Mehran R, Aymong ED, Nikolsky E, Lasic Z, Iakovou I, Fahy M, et al. A simple risk score for prediction of contrast-induced nephropathy after percutaneous coronary intervention: development and initial validation. Journal of the American College of Cardiology. 2004;44(7):1393-1399. https://doi.org/10.1016/j.jacc.2004.06.068

8. Moos S, Nagan G, de Weijert R, van Vemde D, Stoker J, Bipat S. Patients at risk for contrast-induced nephropathy and mid-term effects after contrast administration: a prospective cohort study. Neth J Med. 2014;72(7):363-371.

9. Nash K, Hafeez A, Hou S. Hospital-acquired renal insufficiency. Am J Kidney Dis. 2002;39(5):930-936. https://doi.org/10.1053/ ajkd.2002.32766

10. Moos SI, van Vemde DN, Stoker J, Bipat S. Contrast induced nephropathy in patients undergoing intravenous (IV) contrast enhanced computed tomography (CECT) and the relationship with risk factors: a meta-analysis. Eur J Radiol. 2013;82(9):e387-e399. https://doi.org/10.1016/j.ejrad.2013.04.029

11. Selistre L da S, de Souza VC, Dubourg L, Bernardes Wagner M, Hoefel Filho JR, Saitovitch D. Nefropatia induzida por contraste após tomografia computadorizada. J Bras Nefrol. 2015;37(1):27-31. https://doi.org/10.5935/0101-2800.20150005

12. Rao QA, Newhouse JH. Risk of nephropathy after intravenous administration of contrast material: a critical literature analysis. Radiology. 2006;239(2):392-397. https://doi.org/10.1148/radiol.2392050413

13. Sonhaye L, Kolou B, Tchaou M, Amadou A, Assih K, N'Timon B, et al. Intravenous contrast medium administration for computed tomography scan in emergency: a possible cause of contrast-induced nephropathy. Radiol Res Pract. 2015;2015. https://doi. org $/ 10.1155 / 2015 / 805786$

14. Skarupskiene I, Balciuviene V, Ziginskiene E, Kuzminskis V, Vaiciuniene R, Bumblyte IA. Changes of etiology, incidence and outcomes of severe acute kidney injury during a 12-year period (2001-2012) in large university hospital. Nephrol Ther. 2016;12(6):448453. https://doi.org/10.1016/j.nephro.2016.03.003

15. Herrera R, Orantes CM, Almaguer M, Alfonso P, Bayarre HD, Leyva IM, et al. Características clínicas de la enfermedad renal crónica de causas no tradicionales en las comunidades agrícolas salvadoreñas. MEDICC Rev. 2014;16(2):39-47.

16. Sistema Integrado de Información de la Protección Social-SISPRO (MSPS) (Sistema de Información en Salud del Ministerio de protección Social [Sispro]), cubo cuenta de alto costo. 2015. Consultado el 4 de noviembre de 2017.

17. Almaguer M, Herrera R, Orantes CM. Enfermedad renal crónica de causa desconocida en comunidades agrícolas. MEDICC Rev. 2014;16(2):9-15. 
Rev. Colomb. Nefrol. 2018;5(2): 118 - 126 http://www.revistanefrologia.org http://dx.doi.org/10.22265/acnef.0.0.303

18. Valette X, Parienti JJ, Plaud B, Lehoux P, Samba D, Hanouz JL. Incidence, morbidity, and mortality of contrast-induced acute kidney injury in a surgical intensive care unit: a prospective cohort study. Journal of critical care. 2012;27(3):322.e1-e5. https://doi. org/10.1016/j.jerc.2011.08.005

19. Cely CM, Schein RM, Quartin AA. Risk of contrast induced nephropathy in the critically ill: a prospective, case matched study. Critical Care. 2012;16(2):R67. https://doi.org/10.1186/cc11317

126 Factores asociados a nefropatía inducida por contraste. 